La Revue

des Droits

de l'Homme

\section{La Revue des droits de l'homme}

Revue du Centre de recherches et d'études sur les droits fondamentaux

$11 \mid 2017$

Revue des droits de l'homme - $\mathrm{N}^{\circ} 11$

\title{
La mobilisation de l'égalité formelle contre les mesures tendant à l'égalité réelle entre les femmes et les hommes : le droit de la non-discrimination contre les femmes?
}

\section{Elsa Fondimare}

\section{(2) OpenEdition}

Journals

Édition électronique

URL : http://journals.openedition.org/revdh/2885

DOI : $10.4000 /$ revdh.2885

ISSN : 2264-119X

Éditeur

Centre de recherches et d'études sur les droits fondamentaux

Référence électronique

Elsa Fondimare, "La mobilisation de l'égalité formelle contre les mesures tendant à l'égalité réelle entre les femmes et les hommes : le droit de la non-discrimination contre les femmes ? », La Revue des droits de l'homme [En ligne], 11 | 2017, mis en ligne le 10 janvier 2017, consulté le 08 juillet 2020. URL : http://journals.openedition.org/revdh/2885 ; DOl : https://doi.org/10.4000/revdh.2885

Ce document a été généré automatiquement le 8 juillet 2020 .

Tous droits réservés 


\title{
La mobilisation de l'égalité formelle contre les mesures tendant à l'égalité réelle entre les femmes et les hommes : le droit de la non- discrimination contre les femmes?
}

\author{
Elsa Fondimare
}

1 Dans le rapport du Conseil d'État sur le principe d'égalité de 1996, il est écrit que «la non-discrimination [...] constitue une application stricte du principe d'égalité entre les sexes, affirmé par le préambule de la Constitution de 1946 toujours en vigueur. En revanche, elle ne peut avoir pour prétention de réaliser l'égalité réelle des hommes et des femmes dans tous les aspects de la vie sociale ${ }^{1}$. Cette affirmation constitue une illustration de l'un des nombreux discours juridiques qui érigent une opposition entre égalité formelle et égalité réelle. Une telle opposition conduit à limiter, et parfois même à empêcher, l'appréhension de dispositifs juridiques permettant de lutter concrètement contre les inégalités de fait qui touchent encore majoritairement les femmes.

2 Cette mise en opposition s'explique par les différentes significations et les différents objectifs assignés, d'un côté, à l'égalité formelle et, de l'autre, à l'égalité réelle. L'égalité formelle est, d'une part, communément définie comme exigeant le traitement juridique identique des personnes placées dans des situations similaires². Appliquée à l'égalité des sexes, cette conception implique de traiter de façon identique les femmes et des hommes, considérés comme placés, par principe, dans la même situation. Elle implique donc uniquement l'interdiction de la discrimination directe en raison du sexe par la neutralité des normes juridiques et des pratiques - des employeurs ou des fournisseurs d'accès aux biens et aux services - au regard du sexe des individus. Au contraire, la notion d'égalité «réelle» ou «substantielle» renvoie aux traitements juridiques différenciés entre les femmes et les hommes, autorisés ou imposés afin de remédier aux 
inégalités de situations qui existent entre les femmes et les hommes ${ }^{3}$. A ce titre, le législateur a adopté, dès les années 1980, de nombreux textes prévoyant des mesures positives, «constituant une différenciation juridique de traitement, créée à titre temporaire, dont l'autorité normative affirme expressément qu'elle a pour but de favoriser une catégorie déterminée de personnes physiques ou morales au détriment d'une autre, afin de compenser des inégalités de fait préexistantes entre elles ${ }^{4}$. En droit positif français, des mesures préférentielles prises, pour les mères de famille, en matière de retraite, visent par exemple à compenser les inégalités de fait subies dans le déroulement de leur carrière; de même, des actions positives adoptées en faveur des femmes tendent à instaurer la parité dans les instances politiques ou professionnelles. L'objet de cet article est précisément de s'intéresser à la manière dont a été construite l'opposition entre ces deux interprétations du principe juridique d'égalité : celle qui impose de traiter de façon identique les individus placés dans des situations identiques, et celle qui implique de traiter différemment les individus dans des situations différentes, notamment en raison de leur sexe, afin de remédier aux inégalités de faits ${ }^{5}$.

Une des difficultés principales réside dans le fait que les discours juridiques ne se contentent pas d'opposer ces deux acceptions; ils font également prévaloir l'égalité formelle par rapport à l'égalité réelle. Dès lors, en privilégiant l'égalité formelle, les interprètes de l'égalité ne conduisent pas toujours à favoriser les catégories les plus désavantagées dans les faits, telles que les femmes. C'est le cas, notamment, lorsqu'ils permettent aux hommes, sur le fondement de l'égalité formelle, de remettre en cause les mesures dites de "discriminations positives " visant à corriger ou à compenser les inégalités de fait désavantageant les femmes. Au vu de la construction de cette opposition hiérarchique dans les discours juridiques - en particulier ceux des juges et de la doctrine -, il est donc permis de s'interroger sur le rôle paradoxal joué par le principe d'égalité et le droit de la non-discrimination dans la perpétuation des inégalités entre les femmes et les hommes. Il est en effet paradoxal que l'interprétation du principe juridique d'égalité puisse conduire à limiter des outils tendant à la réalisation concrète de cette égalité.

4 A travers l'analyse des discours des interprètes de l'égalité - principalement des juges -, on relèvera dès lors le caractère systématique de l'opposition entre égalité formelle et égalité réelle, qui a conduit à une interprétation dominante du principe d'égalité, fondée sur des valeurs méritocratiques, aboutissant à limiter les différences de traitement juridiques entre les sexes visant l'égalité réelle. Il s'agit ainsi d'analyser comment l'égalité formelle a été non seulement construite comme une limitation aux mesures positives en faveur des femmes (I), afin de préserver une égalité de type méritocratique (II), mais également comme un obstacle à la consécration d'un « droit à l'égalité réelle » (III).

\section{La construction de l'égalité réelle comme dérogation à l'égalité formelle}

5 Dans son sens formel, le principe d'égalité impose seulement au législateur, à l'administration et à certains acteurs sociaux, de traiter de façon identique les individus placés dans des situations identiques, selon la formulation adoptée par l'article 6 de la Déclaration de 1789 : «La loi est la même pour tous ». Il ne s'agit cependant pas ici d'affirmer que le droit français retient uniquement cette interprétation de 
l'égalité, ni de nier que le droit français a été construit à la fois selon une logique universaliste et différencialiste ${ }^{6}$. Il s'agit simplement de souligner la prééminence, le caractère principiel, dans les discours des juges et de la doctrine, d'une conception de l'égalité privilégiant l'identité de traitement ${ }^{7}$. Celle-ci est en effet considérée comme étant axiologiquement et juridiquement supérieure, le rapporteur public Pellissier évoquant en ce sens un "idéal du traitement identique $»^{8}$. Le Conseil constitutionnel juge ainsi traditionnellement que "le principe d'égalité impose seulement qu'à des situations semblables soient appliquées les mêmes règles " ${ }^{9}$, de même que le rapport public du Conseil d'État de 1996 affirme que "l'universalité de la règle épuise l'égalité » ${ }^{10}$. Cette conception formelle de l'égalité a été appliquée à l'égalité des sexes, concrétisée à travers l'affirmation d'un principe de non-discrimination en raison du sexe, ce qui a mené, tout au long du XXème siècle, à la remise en cause des inégalités juridiques excluant majoritairement les femmes de la jouissance des droits. L'application du principe d'égalité dans un sens formel a ainsi permis d'étendre aux femmes les droits civils ${ }^{11}$ et politiques ${ }^{12}$ réservés jusqu'alors aux hommes. De même, l'émergence d'un principe de non-discrimination en raison $\mathrm{du}$ sexe, sous l'impulsion du droit de l'Union européenne ${ }^{13}$, s'est par exemple manifestée par l'interdiction faite aux employeurs de distinguer les salariés selon le sexe, s'agissant du recrutement ou du salaire. L'interprétation formelle du principe d'égalité des sexes et la consécration d'un principe de non-discrimination en raison du sexe ont donc été des premières étapes nécessaires à l'instauration de l'égalité des droits entre les femmes et les hommes.

6 Mais la consécration d'un principe d'égalité comme exigeant avant tout une indifférence de traitement juridique, a conduit les juges et la majorité de la doctrine à appréhender les différences de traitement comme des ruptures de l'égalité ou comme des dérogations à l'égalité ${ }^{14}$, et ce même lorsque celles-ci ont pour but de lutter contre les inégalités de fait. Le vocabulaire de la «dérogation» est ainsi présent dans la plupart des discours doctrinaux portant sur les mesures positives en faveur de l'égalité réelle entre les femmes et les hommes ${ }^{15}$. Certains auteurs se réfèrent, en ce sens, volontiers à la notion de «discriminations positives" pour désigner ces mesures, considérant avant tout celles-ci comme des ruptures à la conception prééminente du principe d'égalité exigeant l'absence de distinction ${ }^{16}$. La conception des mesures positives comme ruptures ou dérogations au principe d'égalité est par exemple exposée à plusieurs reprises, de façon significative, dans les conclusions du rapporteur public Pellissier sur l'affaire relative à la parité dans les chambres d'agriculture. Celui-ci affirme en effet que: "lorsque ces mesures sont fondées sur le sexe des personnes, qu'elles soient contraignantes - comme dans la technique des quotas - ou incitatives [...], elles se heurtent au principe d'égalité en droit qui implique la suppression dans la norme de tous les marqueurs de genre $»^{17}$. Ces discours prennent ainsi part à la construction d'une prééminence de l'égalité formelle sur l'égalité réelle, en considérant de telles mesures comme des ruptures ou des dérogations à au principe d'égalité.

7 Les juges appréhendent également les différences de traitement, selon une formule récurrente, comme des dérogations possibles au principe juridique d'égalité si elles sont justifiées par un but d'intérêt général ${ }^{18}$, par exemple, lorsqu'elles visent à atteindre une égalité réelle ou de fait. Dans sa décision du 14 août 2003 sur la loi portant réforme des retraites, le Conseil constitutionnel a admis que des avantages sociaux en matière de retraite pouvaient être accordés uniquement aux femmes, car l'intérêt général justifiait une telle dérogation au principe d'égalité ${ }^{19}$. En matière de 
parité en politique, le Conseil constitutionnel a, de la même manière, déclaré conformes à la Constitution les dispositions législatives visant à imposer, pour les élections au scrutin de liste, un nombre égal de candidats de chaque $\operatorname{sexe}^{20}$, tout en rappelant leur caractère dérogatoire. Il a, en ce sens, considéré que « rien ne s'oppose [...] à ce que le pouvoir constituant introduise dans le texte de la Constitution des dispositions nouvelles qui, dans les cas qu'elles visent, dérogent à des règles ou principes de valeur constitutionnelle; qu'il en est ainsi des dispositions précitées qui ont pour objet et pour effet de lever les obstacles d'ordre constitutionnel relevés par le Conseil constitutionnel dans les décisions susmentionnées ${ }^{21}$. L'affirmation par les juges du caractère dérogatoire de ces mesures positives différencialistes participe donc à la construction de l'opposition hiérarchique entre l'égalité formelle et l'égalité réelle.

8 Or, cette opposition hiérarchique a mené les juges - constitutionnels, administratifs et européens - à exercer un contrôle strict des mesures positives. Lors de l'examen de la constitutionnalité des mesures visant à permettre l'égal accès des femmes et des hommes aux mandats électifs et aux responsabilités professionnelles, le Conseil constitutionnel a ainsi jugé que celles-ci étaient contraires au principe d'égalité formelle, issu de l'article 6 de la Déclaration de 1789, et ce à plusieurs reprises en 1982, en 1999, en 2001 et en 2006. Dans les décisions de 2001 et de 2006, le Conseil exerce un contrôle strict des mesures paritaires prises par le législateur, en raison du caractère dérogatoire de ces dernières. Il refuse ainsi que l'alinéa 5 de l'article 3 de la Constitution puisse s'appliquer en dehors des élections à des mandats et fonctions politiques, à la composition du Conseil supérieur de la magistrature et à la composition des conseils d'administration et de surveillance des entreprises ${ }^{22}$. Cette interprétation stricte a été reprise par le Conseil d'État dans ses arrêts de 2013 relatifs à la parité dans les listes pour les élections des chambres d'agriculture et dans les fédérations sportives. Il juge en effet que « le législateur est seul compétent [...] pour adopter les règles destinées à favoriser l'égal accès des femmes et des hommes aux mandats, fonctions et responsabilités mentionnés à l'article 1er précité ${ }^{23}$. Une telle interprétation a abouti à l'annulation des décrets adoptés par le Premier ministre qui prévoyaient, sans habilitation législative, d'introduire des mesures paritaires dans les instances susmentionnées. Le lien entre le caractère dérogatoire des mesures paritaires et la limitation dans leur mise en œuvre est d'ailleurs clairement souligné par le rapporteur public Pellissier dans cette affaire : "Il nous semble qu'en raison tant de l'importance de ces mesures que de leur caractère dérogatoire au principe constitutionnel d'égalité devant la loi, elles font nécessairement partie des règles et principes fondamentaux qu'il appartient au législateur seul de déterminer ${ }^{24}$. La Cour de justice de l'Union européenne estime, dans le même sens, que les dispositions de la directive prévoyant la faculté de mettre en place des mesures de quotas favorisant l'accès des candidats féminins dans les secteurs de l'emploi où les femmes sont sousreprésentées, constituent une dérogation à un droit individuel consacré par la directive - soit l'égalité de traitement - et sont par conséquent « d'interprétation stricte », ce qui justifie la censure de telles mesures positives ${ }^{25}$.

9 Selon cette logique dérogatoire, les mesures positives sont conformes à l'égalité lorsqu'elles sont limitées, notamment dans le temps, et qu'elles sont destinées à compenser - et non à corriger - un désavantage subi. Ainsi, en matière de pension de retraite, le Conseil d'État a jugé dans l'arrêt Quintanel de mars 2015 que les dispositions $\mathrm{du}$ Code des pensions, permettant indirectement aux femmes fonctionnaires ayant eu des enfants de bénéficier d'une bonification de pension par enfant et d'un départ anticipé à la retraite, ne créaient pas une rupture du principe d'égalité des 
rémunérations. Il admet en effet que ces avantages constituent "une compensation partielle et forfaitaire des retards et préjudices de carrière manifestes qui [...] ont pénalisées [les femmes] $\aleph^{26}$. Si la solution du juge semble à première vue privilégier une conception réelle de l'égalité, c'est toutefois seulement parce que ces avantages sont limités, à la fois dans le temps et de par leur relative efficacité. Le Conseil d'État estime en effet, d'une part, que ces avantages ont un caractère "provisoire» et sont appelées à disparaître, puisque la loi de 2003 a maintenu cette bonification uniquement pour les femmes fonctionnaires et militaires mères d'enfants nés avant le $1^{\mathrm{er}}$ janvier 2004. D'autre part, la bonification est légitime car elle "n'a pas pour objet et ne pouvait avoir pour effet de prévenir les inégalités sociales dont ont été l'objet les femmes » mais prévoit une simple compensation " partielle et forfaitaire ». Le Conseil d'État rappelle que c'est « dans ces conditions" que les avantages dont bénéficient indirectement les femmes fonctionnaires sont conformes au principe d'égalité. On peut penser qu'a contrario, une mesure positive illimitée dans le temps et procurant un avantage substantiellement supérieur aurait constitué une rupture d'égalité. Par conséquent, au regard des jurisprudences du Conseil constitutionnel et du Conseil d'État, il semble que les mesures positives respectent le principe d'égalité uniquement lorsque qu'elles sont dépourvues d'une réelle efficacité, ce qui constitue une limite importante à la lutte contre les inégalités de fait.

La hiérarchie mise en place entre ces deux conceptions de l'égalité, au profit du principe d'égalité formelle, s'exprime également par le caractère facultatif des mesures visant l'égalité réelle qui, contrairement au principe d'égalité formelle ne s'imposent pas au législateur et à l'administration. Ces derniers ont seulement la possibilité d'adopter de telles différences de traitement, selon une jurisprudence constante du Conseil constitutionnel et du Conseil d'État ${ }^{27}$. En ce sens, en matière de parité en politique, les constituants ont préféré le terme «favoriser » à celui de "garantir » lors de la révision constitutionnelle de 1999 introduisant l'objectif d'égal accès - et non de parité, impliquant la réalisation d'une égalité numérique - dans la Constitution, laissant ainsi au législateur le choix d'adopter ou non des mesures contraignantes pour réaliser cet objectif. De même, dans le domaine de l'égalité professionnelle, le droit européen prévoit que l'égalité de traitement entre les femmes et les hommes " n'empêche pas un État membre de maintenir ou d'adopter des mesures prévoyant des avantages spécifiques » en faveur du sexe sous-représenté, "pour assurer concrètement une pleine égalité entre hommes et femmes dans la vie professionnelle ${ }^{28}$, ce qui revient à faire de la lutte contre les inégalités de fait une simple faculté pour les États membres.

11 L'opposition hiérarchique entre égalité formelle et égalité réelle repose par conséquent sur un paradoxe : les mesures positives, en ce qu'elles prévoient une distinction fondée sur le sexe, constituent une dérogation au principe d'égalité, alors même qu'elles tendent à l'égalité réelle entre les femmes et les hommes. On retrouve d'ailleurs cette contradiction dans ces propos de Georges Vedel : «Le législateur peut [...], pour combattre les inégalités ou pour des raisons d'intérêt général qu'il juge supérieures, porter de sérieuses atteintes au principe d'égalité $»^{29}$. Or, il peut sembler incohérent d'opposer, de mettre "en conflit deux significations du principe d'égalité que le droit français cherche précisément à conjuguer $\aleph^{30}$. Mais le paradoxe que constitue l'opposition de l'égalité avec elle-même, peut être expliqué par la volonté de sauvegarder une conception méritocratique de l'égalité, et non de tendre absolument à une égalité réelle entre les femmes et les hommes. En d'autres termes, la construction du jeu de "principe/exception " et l'opération de "conciliation» entre ces deux conceptions de l'égalité, sont fondées 
implicitement sur une " hiérarchie axiologique $»^{31}$, attribuant à l'égalité méritocratique une "valeur éthico-politique ${ }^{32}$ plus importante qu'à l'égalité réelle entre les femmes et les hommes.

\section{La prééminence d'une conception méritocratique de l'égalité}

12 La hiérarchie érigée entre l'égalité formelle et les mesures visant l'égalité réelle permet de préserver la logique méritocratique qui sous-tend le principe d'égalité, tel qu'énoncé à l'article 6 de la Déclaration de $1789^{33}$. Les mesures positives visant à lutter contre les inégalités de fait ne peuvent, dès lors, être conciliées avec le principe d'égalité, que lorsqu'elles servent à rétablir cette égalité méritocratique ou " égalité des chances " c'est-à-dire, lorsqu'elles permettent de contrer les inégalités - notamment celles fondées sur le sexe - qui entrave l'accès aux droits et, de là, qui empêche la réussite de chacun selon ses capacités individuelles ${ }^{35}$. En revanche, lorsqu'elles se matérialisent, par des dispositifs correcteurs qui, écartant la question des capacités individuelles, ont pour but l'accession prioritaire des femmes à des lieux de pouvoirs, ou l'imposition d'avantages sociaux aux seules femmes, elles constituent des ruptures d'égalité devant être limitées. En d'autres termes, comme l'affirme Geneviève Koubi, avec l'égalité des chances, «le slogan à chacun son mérite prend le pas sur le moteur de la redistribution sociale ${ }^{36}$. Malgré l'adoption croissante par le législateur de dispositifs visant une égalité réelle ou de résultat ${ }^{37}$, il apparait ainsi que la lutte contre les inégalités de fait touchant majoritairement les femmes constitue, dans le discours des juges tout du moins, un objectif de moindre importance que la préservation d'une égalité méritocratique.

Cette prépondérance de l'égalité méritocratique sur l'égalité réelle est significative dans la jurisprudence constitutionnelle et administrative contrôlant les mesures visant l'égal accès des femmes et des hommes à des fonctions électives ou à des responsabilités professionnelles. Ainsi, dans une décision de 2002, le Conseil constitutionnel juge conforme à l'article 6 de la Déclaration de 1789 les dispositions de la loi de modernisation sociale fixant un simple objectif de représentation équilibrée entre les femmes et les hommes dans la composition des jurys de concours, sous réserve de ne pas faire "prévaloir, lors de la constitution de ces jurys, la considération du genre sur celles des compétences, des aptitudes et des qualifications $»^{38}$. Dans l'arrêt Lesourd de 2007, le Conseil d'État reprend ce raisonnement, en jugeant que le décret du 3 mai 2002 fixant une proportion minimale d'un tiers de personnes de chaque sexe dans les jurys de concours de recrutement des fonctionnaires d'État, ne méconnaît pas les exigences de l'article 6, car il se «borne à imposer à l'administration de prendre en compte l'objectif de représentation équilibrée entre les femmes et les hommes et ne saurait avoir pour objet ou pour effet, pour la composition des jurys, de fixer une proportion de personnes de chaque sexe qui s'imposerait à peine d'irrégularité de concours ${ }^{39}$. Ainsi, en mentionnant la nécessité d'un certain degré de compétence pour tous les membres du jury de concours, le décret entend continuer à faire prévaloir le critère du mérite sur celui du sexe et réduit ses dispositions à de simples objectifs sans quotas contraignants. En revanche, dans une décision de 2006, la primauté de l'égalité méritocratique conduit le Conseil constitutionnel à déclarer contraires à la Constitution les mesures imposant le respect de proportions déterminées entre les femmes et les hommes au sein des conseils d'administration et de surveillance des entreprises. En effet, ces mesures constituent 
des règles contraignantes fondées sur le sexe des personnes et par conséquent, elles font « prévaloir la considération du sexe sur celles des capacités et de l'utilité commune »"

Il convient de noter que les juges constitutionnels et administratifs se fondent ici sur l'article 6 de la Déclaration de 1789. Ainsi, ces mesures paritaires, en instituant une différence de traitement entre les femmes et les hommes, heurtent non pas l'égalité entre les hommes et les femmes, dans son sens formel, mais le principe selon lequel « tous les citoyens [...] sont également admissibles à toutes dignités, places et emplois publics, selon leur capacité, et sans autre distinction que celle de leurs vertus et de leurs talents ». C'est donc bien parce que ces mesures empêchent un individu d'accéder à un poste de pouvoir sur le critère de sa compétence et de son mérite, qu'elles constituent une rupture d'égalité, et non pas parce que elles violent la règle de non-discrimination entre les femmes et les hommes. Là encore, le principe de non-discrimination en raison du sexe semble être un objectif secondaire par rapport à l'égalité méritocratique.

Les discours des interprètes de l'égalité font ainsi primer l'aspect individuel du principe d'égalité - qui implique que chacun ne puisse être traité différemment qu'au regard de ses compétences et de son mérite personnel - sur la lutte contre les inégalités de fait et de droit - touchant majoritairement les femmes. Le rapport du Conseil d'État de 1996 fait clairement mention des conceptions communautaire et française de l'égalité, qui placent «en premier le droit individuel à l'égalité et en second la dimension correctrice, compensatrice d'effets préjudiciables résultant des comportements de la société à l'égard des femmes $»^{41}$. Une telle hiérarchisation explique pourquoi les recours exercés par des hommes, tendant à remettre en cause des "discriminations positives" en faveur des femmes, sont accueillis aussi bien par les juges français que par les juges européens. De telles « discriminations positives ", "à rebours ", à l'encontre des hommes, constituent en effet pour les juges des différences de traitement illégitimes, car elles font primer l'aspect correcteur de la lutte contre les inégalités de fait sur le droit individuel des hommes à être traité uniquement sur le critère du mérite.

Dans le domaine de l'emploi, la Cour de justice de l'Union européenne juge ainsi qu'une "réglementation nationale qui garantit la priorité absolue et inconditionnelle aux femmes lors d'une nomination ou d'une promotion va au-delà d'une promotion de l'égalité des chances et dépasse les limites de l'exception prévue» par la directive ${ }^{42}$. Dès lors, pour la Cour, la considération du sexe ne doit pas être prise en compte, pour instaurer un quota, si les qualifications entre les candidats sont inégales, car cela revient à faire prévaloir le critère du sexe sur celui du mérite. Ceci constitue par conséquent une discrimination à l'encontre du candidat masculin ${ }^{43}$. C'est donc bien l'égalité des chances, qui permet de compenser les discriminations passées en rétablissant l'égalité entre hommes et femmes à compétence égale, qui est privilégiée par la Cour, et non l'égalité de résultat, qui tend à attribuer automatiquement des postes de travail aux femmes pour atteindre une égalité numérique et corriger ainsi les inégalités de fait ${ }^{44}$. L'égalité des chances préserve ainsi le droit individuel (des hommes) à n'être distingués que selon leurs compétences, puisque seules les mesures positives prévoyant des «clauses d'ouverture $\|^{45}$, c'est-à-dire le recrutement des hommes lorsque ceux-ci sont plus compétents, sont autorisées. Si la Cour de justice admet donc - de manière de plus en plus souple ${ }^{46}$ - les «actions positives » en faveur des femmes, elle proscrit cependant les « discriminations positives» qui portent atteinte à la logique méritocratique ${ }^{47}$.

La prééminence accordée par les juges à l'égalité formelle sur l'égalité réelle n'a donc pas exactement pour objet de préserver une identité de traitement entre les individus. 
Elle tend davantage à sauvegarder le principe posé à l'article 6 de la Déclaration de 1789 selon lequel les distinctions ne peuvent être fondées que sur les capacités, le mérite et les talents. Les mesures positives en faveur des femmes ne sont dès lors légitimes, conciliables avec le principe d'égalité, que lorsqu'elles visent à rétablir les mêmes chances entre les femmes et les hommes, et non la garantie effective des mêmes droits. C'est par conséquent moins l'égalité réelle et la lutte contre les inégalités de fait touchant majoritairement les femmes qui importent, dans les discours et décisions étudiés, que la sauvegarde d'une égalité de type libérale ${ }^{48}$ préservant les différences de traitement fondée sur le mérite ${ }^{49}$. L'égalité réelle entre les femmes et les hommes apparaît donc comme axiologiquement inférieure à l'égalité méritocratique dans le discours des interprètes de l'égalité. Cette infériorité axiologique s'est également traduite sur le plan juridique par l'absence de consécration de l'égalité réelle comme droit individuel.

\section{L'égalité formelle contre la consécration de l'égalité réelle comme un droit de l'homme} à la fois comme "un droit fondamental en soi » et comme "une condition d'exercice des autres droits $\|^{50}$. Il figure du reste parmi les droits les plus invoqués dans le cadre du contrôle de constitutionnalité des lois ${ }^{51}$. La reconnaissance du principe d'égalité comme droit fondamental ne semble toutefois valoir que lorsqu'il est appréhendé dans un sens formel. L'égalité réelle n'est en effet pas reconnue comme un droit, mais comme un simple objectif non-contraignant pour le législateur. La disposition constitutionnelle prévoyant l'égal accès des femmes et des hommes aux mandats et fonctions politiques et aux responsabilités professionnelles, à savoir l'alinéa 2 de l'article $1^{\text {er }}$ de la Constitution, ne proclame ainsi, pour reprendre les mots de Gilles Pellissier, «aucun droit, mais confère à un pouvoir normatif une faculté d'opérer des différences de traitement pour réaliser une égalité réelle $»^{52}$.

L'absence de reconnaissance d'un droit à l'égalité réelle est particulièrement significative dans la solution retenue par le Conseil constitutionnel dans sa décision QPC du 24 avril 2015, relative à la parité dans les conseils académiques des universités ${ }^{53}$. Dans cette affaire, les deux conceptions de l'égalité se confrontent frontalement dans les requêtes des requérants et des intervenants. Le requérant, le Conseil des présidents, faisait notamment valoir que les mesures paritaires violaient le principe d'égalité devant la loi, en conduisant à évincer des candidats du Conseil académique restreint pour des considérations tenant à leur sexe. Au contraire, les intervenants, un groupe d'enseignants-chercheurs, défendait plutôt une conception réelle de l'égalité, en soutenant, sur le fondement de l'incompétence négative, que les mesures prises par le législateur méconnaissaient le principe de parité. Ils et elles soutenaient que ce principe aurait dû conduire à instaurer la parité pour les formations du Conseil académique compétentes pour les questions relatives à tous les enseignantschercheurs, y compris pour celles relatives aux professeurs, ce que ne prévoyait pas le Code de l'éducation ${ }^{54}$. La solution retenue par le Conseil est pourtant à première vue plutôt favorable à la parité et, partant, à l'égalité réelle. Le Conseil constitutionnel a en effet estimé que le législateur avait légitimement assuré la conciliation entre la parité et l'égalité et il a, par conséquent, rejeté la demande des requérants. Il a cependant 
également rejeté le grief invoqué par les intervenants. Tout en reconnaissant que l'alinéa 2 de l'article $1^{\text {er }}$ de la Constitution confère au législateur le pouvoir de prendre des mesures paritaires incitatives ou contraignantes, il considère néanmoins que « cette disposition n'institue pas un droit ou une liberté que la Constitution garantit » et que "sa méconnaissance ne peut donc être invoquée à l'appui d'une question prioritaire de constitutionnalité $\aleph^{55}$. Dès lors, à travers le rejet de la parité comme droit fondamental, le Conseil constitutionnel empêche d'appréhender l'égalité réelle comme un droit individuel, susceptible d'être invoqué par des requérants, notamment à l'occasion d'une question prioritaire de constitutionnalité. Ainsi, ni la disposition constitutionnelle relative à la parité, ni l'alinéa 3 du Préambule de 1946 sur l'égalité des droits, ne constituent un fondement juridique pour un droit à l'égalité réelle, qui demeure une simple faculté pour le législateur.

Par conséquent, la construction de la prééminence d'une conception formelle de l'égalité préservant la méritocratie n'a pas seulement abouti à limiter les mesures tendant à l'égalité réelle. Elle conduit, bien plus, à empêcher la consécration de celle-ci comme un droit individuel, qui serait invocable par les individus pour contester l'action, ou plutôt l'inaction du législateur et des pouvoirs publics, dans la lutte contre les inégalités de fait, dans les domaines où elles désavantagent encore majoritairement les femmes. S'agissant du principe d'égalité, le phénomène de "droit de l'homme contre lui-même" ne saurait donc être compris simplement dans le sens d'une opposition entre deux conceptions contraires de l'égalité, qui entraîne la prééminence axiologique et juridique de l'une sur l'autre. Le principe d'égalité constitue également " un droit de l'homme contre lui-même ", en ce que la prédominance d'une conception formelle de l'égalité empêche la reconnaissance même de l'égalité réelle en tant que droit.

21 La prééminence, dans les discours juridiques dominants, d'une certaine conception dite «républicaine» de l'égalité, conduit donc significativement à limiter les outils juridiques visant l'égalité réelle. Le caractère "principiel » de l'égalité formelle empêche d'appréhender ces outils autrement que comme une dérogation à l'égalité et une forme de discrimination. Pourtant, d'autres instruments juridiques envisagent ces outils comme des moyens permettant de réaliser le principe d'égalité, et non comme des ruptures de ce dernier ; c'est le cas de la Convention pour l'élimination de toutes les formes de discriminations à l'égard des femmes, dont l'article 4 dispose que «l'adoption par les États parties de mesures temporaires spéciales visant à accélérer l'instauration d'une égalité de fait entre les hommes et les femmes n'est pas considérée comme un acte de discrimination». On peut ainsi imaginer que la compréhension hiérarchique et conflictuelle des différentes interprétations de l'égalité laisse place à une compréhension pluraliste, plus complexe, des différents sens de l'égalité mis au même niveau: dans une perspective pragmatique, les différents sens de l'égalité seraient alors, selon les contextes, tous nécessaires à la réalisation de l'égalité hommesfemmes ${ }^{56}$.

Il n'en demeure pas moins que le principe d'égalité demeure construit en droit français comme un "droit de l'homme contre lui-même». Cette opposition joue contre les catégories les plus désavantagées, car elle limite la prise en compte juridique d'outils de lutte contre les inégalités de fait structurelles. Il convient dès lors de ne pas se reposer sur les acquis de l'égalité formelle et du droit de la non-discrimination, qui empêchent 
de s'interroger sur la façon dont la construction de ces principes contribue à perpétuer les subordinations actuelles ${ }^{57}$.

\section{NOTES}

1. Rapport du Conseil d'État, Sur le principe d'égalité, Paris, La documentation française, 1998, p. 105.

2. BYRNES Andrew, "Article 1 », in FREEMAN Marsha, CHINKIN Christine, RUDOLF Beate, The UN Convention on the elimination of all forms of discrimination against women. A Commentary, Oxford University Press, 2012, p. 53 : “'Formal equality' often refers to [...] the 'samenesse', 'similarly situated', or 'identical treatment' approach to equality. It embodies the presumption that equality means that all persons are to be treated identically, and a failure to do so amounts to discrimination or a denial of equality".

3. RAMBAUD Patrick, «L'égalité des sexes en droit social communautaire », Recueil Dalloz, 1998, p. 111 : «Ambivalente, la notion englobe à la fois l'égalité formelle qui, à la manière d'un principe supérieur de l'égalité impose au législateur de n'édicter que des normes neutres, des normes indifférentes au sexe des travailleurs dont elles aménagent la protection, et au juge de censurer toute atteinte à cette neutralité, et l'égalité réelle ou substantielle qui, en tant que principe d'action, pousse au contraire le législateur, sous la pression de l'évolution sociale, à prendre des mesures en faveur du sexe jugé défavorisé ".

4. LANQUETIN Marie-Thérèse, «La double discrimination à raison du sexe et de la race ou de l'origine ethnique : approche juridique ", Synthèse du Rapport Final Convention de recherche FASILD / Service des Droits des Femmes Ministère de l'emploi et de la Solidarité, décembre 2002, http:// www.association-alda.org/attachments/705_Lanquetin_02.pdf.

5. MELIN-SOUCRAMANIEN Ferdinand, Le principe d'égalité dans la jurisprudence du Conseil constitutionnel, Paris, Economica, 1997, p. 20: «La distinction cardinale dans ce domaine consiste à différencier l'égalité de droit et l'égalité de fait. On oppose aussi, mais cela revient pratiquement au même, l'égalité formelle et l'égalité réelle, l'égalité des chances à l'égalité de résultats, l'égalité globale à l'égalité marginale ou encore, l'égalité civile à l'égalité sociale ».

6. BUI-XUAN Olivia, Le droit public français entre universalisme et différencialisme, Paris, Economica, 2004.

7. JOUANJAN Olivier, "Egalité ", in ALLAND Denis, RIALS Stéphane, Dictionnaire de la culture juridique, Paris, PUF, 2003, p. 586 : "C'est cette idée d'universalité que l'on retrouve clairement dans l'interprétation que donnent généralement les juridictions au principe d'égalité [...] Il n'y a pas d'obligation de différencier les traitements mais seulement celle de traiter de la même manière ce qui est essentiellement semblable ».

8. Conclusions de M. Gilles PELLISSIER, rapporteur public, sous l'arrêt du CE, 7 mai 2013, $n^{\circ} 362280$.

9. Conseil constitutionnel, décision $n^{\circ} 80-128$ DC du 21 janvier 1981 sur la loi relative au travail à temps partiel, considérant 4.

10. Rapport du Conseil d'État, Sur le principe d'égalité, op. cit.

11. On peut citer par exemple la loi du 13 juillet 1965 portant réforme des régimes matrimoniaux, qui accorde, sans distinction de sexe, la pleine capacité de droit aux deux époux. 
12. Article 17 de l'ordonnance du 21 avril 1944: "Les femmes sont électrices et éligibles dans les mêmes conditions que les hommes ".

13. Article 2-1 de la directive 76/207/CEE du Conseil, du 9 février 1976, relative à la mise en œuvre du principe de l'égalité de traitement entre hommes et femmes en ce qui concerne l'accès à l'emploi, à la formation et à la promotion professionnelles, et les conditions de travail : «Le principe de l'égalité de traitement au sens des dispositions ci-après implique l'absence de toute discrimination fondée sur le sexe, soit directement, soit indirectement par référence, notamment, à l'état matrimonial ou familial ».

14. RIVERO Jean, «Rapport sur les notions d'égalité et de discrimination en droit public français ", ", in Les notions d'égalité et de non-discrimination en droit interne et en droit international, Travaux de l'Association Henri Capitant, tome XIV, Paris, Dalloz, p. 350: «Certaines des applications de l'égalité, en droit public, se sont donc écartées de cette abstraction : s'engageant dans la voie de l'égalité réelle, législateur et juge ont différencié les règles selon les situations qu'il s'agissait de régir [...] Dans cette voie, cependant, on s'écarte résolument de l'égalité juridique, symbolisée, et réalisée, par la généralité de la règle ».

15. Voir par exemple: VERKINDT Pierre-Yves, «L'égalité de rémunération entre les hommes et les femmes », Droit social, 2008, p. 1051 : «En matière de lutte contre les discriminations fondées sur le sexe, une place - certes timide - est laissée à la rupture de l'égalité formelle par l'admission de mesures de rattrapage ou de compensation "; HAQUET Thomas, "L'action positive, instrument de l'égalité des chances entre hommes et femmes », RTDE, 2001, p. $305:$ : La jurisprudence sur les actions positives se fonde donc sur une dérogation au principe de l'égalité formelle»; FITTE-DUVAL Annie, "Droit des pensions : l'égalité des sexes à marche forcée? ", AJFP, 2002, p. 4 : "Le principe demeure celui de la recherche de la stricte égalité de traitement qui n'admet que des dérogations justifiées par une différence objective de situation»; JOUANJAN Olivier, «Egalité », op. cit., p. 589 : «Ce principe subit cependant désormais des dérogations qui vont jusqu'à l'altération au nom de la reconnaissance des particularismes de certains groupes»; FAVOREU Louis, "L'inconstitutionnalité des quotas par sexe (sauf pour les élections politiques)», AJDA, 2003, p. 313 : «L'alinéa 4 de l'article 3 C introduit simplement une exception à la norme constitutionnelle générale selon laquelle, aux termes de l'article 6 de la Déclaration des droits de l'homme, 'tous les citoyens sont également admissibles à toutes dignités, places et emplois publics selon leur capacité et sans autre distinction que celles de leurs vertus et de leurs talents' "; MELINSOUCRAMANIEN Ferdinand, Le principe d'égalité dans la jurisprudence du Conseil constitutionnel, op. cit., p. 325 : « En dépit du caractère certainement louable de ces tentatives de faire valoir son altérité, il ne faut pas perdre de vue qu'elles constituent autant de brèches dans l'universalisme à la française ".

16. Voir par exemple : MELIN-SOUCRAMANIEN Ferdinand, Le principe d'égalité dans la jurisprudence du Conseil constitutionnel, op. cit., p. 137: «La discrimination positive qu'elle entendait établir [...] constituait une discrimination fondée sur le sexe expressément interdite par la constitution »; LEVADE Anne, «Discrimination positive et principe d'égalité en droit français », Pouvoirs, ${ }^{\circ} 111,2004 / 4$ : "Le principe constitutionnel d'égalité 'à la française' interdit au législateur de développer une politique de discrimination positive »; JOUANJAN Olivier, « Egalité », op. cit., p. 588 : « La discrimination positive est donc, dans son concept, une véritable discrimination en tant qu'elle renvoie l'individu à un statut, le rabat sur une appartenance qu'il n'a pas choisie (son sexe, sa couleur de peau, son handicap) »; GESLOT Christophe, "Égalité devant la loi et discriminations positive », AJDA, 2006, p. 1961 : « Les mesures de différenciation ainsi prises peuvent être qualifiées de 'discriminations positives' en ce sens que la rupture de l'égalité devant la loi (discrimination) tend vers une plus grande égalité de fait »; CALVES Gwenaële, La discrimination positive, Paris, PUF, 2010.

17. Conclusions de Gilles PELLISSIER, rapporteur public, sous l'arrêt du Conseil d'État du 7 mai $2013, \mathrm{n}^{\circ} 362280$.

18. Conseil constitutionnel, décision $n^{\circ} 97-388$ DC du 20 mars 1988, considérant 27 : « Le principe d'égalité ne s'oppose ni à ce que le législateur règle de façon différente des situations différentes, ni à ce qu'il déroge à l'égalité pour des raisons d'intérêt général, pourvu que, dans l'un et l'autre cas, la différence 
de traitement qui en résulte soit en rapport direct avec l'objet de la loi qui l'établit »; CE, 15 mai 2000, Barroux, n² 200903 : "Considérant que le principe général d'égalité ne s'oppose pas à ce que l'autorité investie du pouvoir réglementaire règle de façon différente des situations différentes ni à ce qu'elle déroge à l'égalité pour des raisons d'intérêt général pourvu que dans l'un comme dans l'autre cas, la différence de traitement qui en résulte soit en rapport avec l'objet de la norme qui l'établit ».

19. Conseil constitutionnel, décision $n^{\circ} 2003-483$ DC du 14 août 2003, considérant 23: " Considérant que le principe d'égalité ne s'oppose ni à ce que le législateur règle de façon différente des situations différentes, ni à ce qu'il déroge à l'égalité pour des raisons d'intérêt général pourvu que, dans l'un et l'autre cas, la différence de traitement qui en résulte soit en rapport direct avec l'objet de la loi qui l'établit; considérant que l'attribution d'avantages sociaux liés à l'éducation des enfants ne saurait dépendre, en principe, du sexe des parents; considérant, toutefois, qu'il appartenait au législateur de prendre en compte les inégalités de fait dont les femmes ont jusqu'à présent été l'objet [...] qu'en raison de l'intérêt général qui s'attache à la prise en compte de cette situation [...] le législateur pouvait maintenir, en les aménageant, des dispositions destinées à compenser des inégalités normalement appelées à disparaître ».

20. Conseil constitutionnel, décision $n^{\circ} 2000-429$ DC du 30 mai 2000, considérant 7 .

21. Ibid., considérant 6 .

22. Conseil constitutionnel, décision $n^{\circ} 2001-445$ DC du 19 juin 2001, considérant 57 et décision $\mathrm{n}^{\circ}$ 2006-533 DC du 16 mars 2006, considérant 14: «Si, aux termes des dispositions du cinquième alinéa de l'article 3 de la Constitution, dans leur rédaction issue de la loi constitutionnelle $n^{\circ} 99-569$ du 8 juillet 1999: 'La loi favorise l'égal accès des femmes et des hommes aux mandats électoraux et fonctions électives', il résulte tant des travaux parlementaires ayant conduit à leur adoption que de leur insertion dans ledit article que ces dispositions ne s'appliquent qu'aux élections à des mandats et fonctions politiques ".

23. CE, 7 mai 2013, $n^{\circ} 362280$; CE, 10 octobre 2013, n’ 359219.

24. Conclusions de Gilles PELLISSIER, op. cit.

25. CJCE, 17 octobre 1995, Eckhard Kalanke c/ Freie Hansestadt Bremen, C-450/93.

26. CE, Ass., 27 mars 2015, Quintanel, $n^{\circ} 372426$, considérant $4:$ : Si, pendant son congé de maternité, la femme fonctionnaire ou militaire conserve légalement ses droits à avancement et à promotion et qu'ainsi la maternité est normalement neutre sur sa carrière, il ressort néanmoins de l'ensemble des pièces produites devant le juge du fond et des données disponibles qu'une femme ayant eu un ou plusieurs enfants connaît, de fait, une moindre progression de carrière que ses collègues masculins et perçoit en conséquence une pension plus faible en fin de carrière ».

27. Conseil constitutionnel, décision $n^{\circ} 87-232$ DC du 7 janvier 1988, considérant 10 : « Le principe d'égalité ne s'oppose ni à ce que le législateur règle de façon différente des situations différentes ni à ce qu'il déroge à l'égalité pour des raisons d'intérêt général pourvu que, dans l'un et l'autre cas, la différence de traitement qui en résulte soit en rapport avec l'objet de la loi qui l'établit »; CE, 15 mai 2000, M. Barroux, $n^{\circ}$ 200903: "Le principe général d'égalité ne s'oppose pas à ce que l'autorité investie du pouvoir réglementaire règle de façon différente des situations différentes ni à ce qu'elle déroge à l'égalité pour des raisons d'intérêt général pourvu que dans l'un comme dans l'autre cas, la différence de traitement qui en résulte soit en rapport avec l'objet de la norme qui l'établit ».

28. Article 2-1 de la directive 76/207/CEE du Conseil, du 9 février 1976.

29. VEDEL Georges, "L'égalité ", in La déclaration des droits de l'homme et du citoyen de 1789, ses origines, sa pérennité, La documentation française, 1990, p. 180.

30. ROMAN Diane, HENNETTE-VAUCHEZ Stéphanie, «Seul le législateur peut imposer la parité hommes-femmes dans les listes de candidats aux élections aux chambres d'agriculture ", RFDA, 2013, p. 882.

31. GUASTINI Riccardo, «Lex superior. Pour une théorie des hiérarchies normatives », Revus, 21, 2013, pp. 47-55. 
32. GUASTINI Riccardo, «Les principes de droit en tant que source de perplexité théorique », in CAUDAL Sylvie, Les principe en droit, Paris, Economica, 2008, p. 118 : « Une hiérarchie axiologique est une relation de valeur créée (non pas par le droit lui-même, comme la hiérarchie des sources mais) par le juge, au moyen d'un jugement comparatif de valeur [...]. Établir une hiérarchie axiologique consiste donc à attribuer à l'un des deux principes en conflit un 'poids' - une 'importance' éthico-politique - majeur par rapport à l'autre ».

33. OZOUF Mona, «Égalité », in Furet François, OZOUF Mona, Dictionnaire critique de la Révolution française, Champs Flammarion, 1992, p. 144.

34. L'égalité des chances peut être définie comme: "La faculté pour les individus de participer, à égalité les uns avec les autres, au jeu social pour l'obtention de biens valorisés suffisamment rares pour qu'ils ne soient pas disponibles pour tous. Bref, la chance c'est ici avant tout 'la possibilité de s'élever à un niveau supérieur dans la société stratifiée » (POIRMEUR Yves, «Le double jeu de la notion d'égalité des chances ", in KOUBI Geneviève, GUGLIELMI Gilles, L'égalité des chances. Analyses, évolutions, perspectives, Paris, La découverte, 2000, p. 93).

35. KOUBI Geneviève, "Vers l'égalité des chances : quelles chances pour le droit? ", in KOUBI Geneviève, GUGLIELMI Gilles, L'égalité des chances. Analyses, évolutions, perspectives, op. cit., p. 82.

36. KOUBI Geneviève, « Vers l'égalité des chances : quelles chances pour le droit ? », op. cit., p. 82. 37. On peut évoquer par exemple la loi $n^{\circ} 2011-103$ du 27 janvier 2011 et la loi $n^{\circ} 2012-347$ du 12 mars 2012 qui ont instauré des quotas par sexe contraignants pour la composition des conseils d'administration et de surveillance des grandes entreprises privées et publiques. La loi $n^{\circ}$ 2014-873 du 4 août 2014 pour l'égalité réelle entre les femmes et les hommes a quant à elle renforcé et étendu les dispositifs paritaires, dans une optique d'égalité de résultat.

38. Conseil constitutionnel, $n^{\circ}$ 2001-455 DC du 12 janvier 2002, considérant 115.

39. CE, 22 juin 2007, Lesourd, $n^{\circ}$ 288206, confirmé par l'arrêt Marchand du 16 mars 2011, $n^{\circ} 337265$.

40. Conseil constitutionnel, $n^{\circ} 2006-533$ DC du 16 mars 2006, considérant 15.

41. Rapport du Conseil d'État sur le principe d'égalité, Paris, La documentation française, 1998, p. 106.

42. CJCE, 17 octobre 1995, Eckhard Kalanke c/ Freie Hansestadt Bremen, C-450/93 ; CJCE, 11 novembre 1997, Hellmut Marschall c/ Land Nordrhein-Westfalen, C-409/95.

43. CJCE, 6 juillet 2000, Katarina Abrahamsson, Leif Anderson et Elisabet Fogelqvist, C-407/98.

44. Conclusions de l'avocat général TESAURO présentées le 6 avril 1995, affaire C-450-93, point 22 : «Les observations qui précèdent confirment bien que l'article 2, paragraphe 4, de la directive permet uniquement d'éliminer les disparités de fait dont souffrent les femmes, mais certainement pas au moyen de discriminations à rebours pures et simples, c'est à dire au moyen de mesures qui ne sont absolument pas destinées à éliminer les obstacles empêchant les femmes d'atteindre les mêmes résultats dans des conditions égales, mais qui visent à leur attribuer directement les résultats eux-mêmes ou, tout au moins, à leur accorder la priorité pour les atteindre uniquement parce qu'elles sont des femmes ».

45. Affaire Marschall précitée, point 24 : «Les femmes ne doivent pas être promues par priorité si des motifs tenant à la personne d'un candidat masculin font pencher la balance en sa faveur ».

46. CJCE, 28 mars 2000, Georg Badeck e.a. c/ Landesanwalt beim Staatsgerichtshof des Landes Hessen, C-158/97.

47. BUI-XUAN Olivia, Le droit public français entre universalisme et différencialisme, op.cit., p. 229 et $\mathrm{s}$.

48. KOUBI Geneviève, "Vers l'égalité des chances : quelles chances pour le droit? ", in KOUBI Geneviève, GUGLIELMI Gilles, L'égalité des chances. Analyses, évolutions, perspectives, Paris, La découverte, 2000, p. 80 : «Les chances s'instituent ainsi dans des rapports reconstruits par la pensée libérale entre droit et économie. Le discours juridique s'incline devant la décision économique. L'égalité des chances est donc un des outils dont disposent les gouvernants pour justifier les distinctions et entériner les conséquences de leurs politiques économiques ».

49. LANQUETIN Marie-Thérèse, «De l'égalité des chances », Droit social, 1996, p. 494. 
50. MELIN-SOUCRAMANIEN Ferdinand, «Le principe d'égalité dans la jurisprudence du Conseil constitutionnel. Quelles perspectives pour la question prioritaire de constitutionnalité ? », Cahiers du Conseil constitutionnel, $\mathrm{n}^{\circ} 29$, octobre 2010.

51. Ibid. : «Dans près d'une décision sur deux rendue par le Conseil constitutionnel dans le cadre du contrôle a priori de l'article 61 de la Constitution, il est fait application du principe d'égalité ». 52. Conclusions de M. Gilles PELLISSIER, op. cit.

53. Conseil constitutionnel, décision $n^{\circ}$ 2015-465 QPC du 24 avril 2015.

54. Article 50 de la loi $n^{\circ} 2013-660$ du 22 juillet 2013 relative à l'enseignement supérieur et la recherche, modifiant l'article L. 712-6-1 du Code de l'éducation.

55. Conseil constitutionnel, décision $n^{\circ}$ 2015-465 QPC du 24 avril 2015.

56. FREDMAN Sandra, «Beyond the dichotomy of formal and substansive equality : towards a new definition of equal rights ", in BOEREFIJN, Temporary special measures. Accelerating de facto equality of women under Article 4(1) UN Convention on the elimination of all forms of discrimination against women, Antwerp, Oxford, New york, Intersentia, $2003:$ : In some cases, [differentiation] would become irrelevant, in other cases, the differences that exists between men and women would be appropriately recognized and valued in social arrangement $»$.

57. SIEGEL Reva, «Why equal protection no longer protect: the evolving forms of statusenforcing state action », 49 Stanford Law Review, 1996-1997, p. 1111.

\section{RÉSUMÉS}

Les juges français, comme la doctrine, opposent traditionnellement différentes interprétations du principe d'égalité des sexes, l'égalité formelle et l'égalité réelle. Or, cette mise en opposition conduit à faire du principe d'égalité un «droit de l'homme contre lui-même »: le principe d'égalité, dans son sens formel, est précisément mobilisé dans les discours juridiques, comme limite aux dispositifs visant l'égalité réelle, telles que les actions positives en faveur de l'accès des femmes à certaines fonctions ou emplois publics. Plus encore, la prédominance axiologique du principe d'égalité formelle aboutit à empêcher la reconnaissance d'un « droit à l'égalité réelle ». Une telle hiérarchisation entre les différentes significations du principe d'égalité vise ainsi à préserver la prédominance d'une conception méritocratique de l'égalité.

French judges and legal literature traditionally oppose different interpretations of sex equality principle, formal equality and substantive equality. But this opposition leads to consider the equality principle as a "human right against itself" : the equality principle, in a formal sense, appears in legal discourses as a limitation to substantive equality's mechanisms, such as positive actions in favour of women's access to certain public functions or employments. Moreover, the axiological predominance of the equality principle in its formal sense prevents from recognizing a "right to substantive equality". Such a hierarchy between the different significations of the equality principle aims to preserve the predominance of a conception of equality based on meritocracy. 
INDEX

Mots-clés : égalité formelle, égalité réelle, égalité des sexes, non-discrimination, parité, action positive

Keywords : formal equality, substantive equality, sex equality, anti-discrimination, parity, positive action

\section{AUTEUR}

\section{ELSA FONDIMARE}

Elsa Fondimare est attachée temporaire d'enseignement et de recherche à l'Université Paris Ouest Nanterre La Défense, doctorante au CREDOF. 